\title{
Laser-induced surface acoustic waves: An alternative method to nanoindentation for the mechanical characterization of porous nanostructured thin film electrode media
}

\author{
Gabriel Chow ${ }^{1}$, Evan Uchaker $^{2}$, Guozhong $\mathrm{Cao}^{2}$, Junlan Wang ${ }^{1 \neq}$ \\ ${ }^{1}$ Department of Mechanical Engineering, University of Washington, Seattle, WA 98195, USA \\ ${ }^{2}$ Department of Materials Science and Engineering, University of Washington, Seattle, WA 98195, USA \\ ‡ Corresponding Author, Email: junlan@uw.edu, Phone: (206) 543 - 4601
}

\begin{abstract}
The mechanical characterization of electrode materials in thin film lithium ion batteries is currently a sparse area. However, mechanical studies could offer valuable insight since the performance and breakdown of active materials is electromechanically coupled. In this paper, a porous nanostructured $\mathrm{V}_{2} \mathrm{O}_{5}$ cathode thin film with demonstrated high electrochemical performance was investigated by a laser-induced surface acoustic wave technique (LiSAW) that mitigates some of the challenges associated with the popular nanoindentation technique. The intent was to explore the capability of LiSAW in measuring the elastic modulus of the nanostructured film such that a reliable methodology could be produced to mechanically characterize challenging electrode materials. LiSAW measured a modulus of $53 \pm 4 \mathrm{GPa}$ for the porous $\mathrm{V}_{2} \mathrm{O}_{5}$ film and had no problems coping with the $40 \mathrm{~nm}$ roughness and delicate structure. On the other hand, nanoindentation produced a modulus of $50 \pm 10 \mathrm{GPa}$, which is comparable to LiSAW, but with considerably higher uncertainty from roughness. For porous nanostructured electrodes, and other challenging films, that are too soft, thin, or delicate for traditional nanoindentation measurements, LiSAW is a potentially excellent alternative. LiSAW testing on many other electrode materials would be instrumental in developing a better understanding between the mechanical and electrochemical properties of thin film battery materials.
\end{abstract}

Keywords: mechanical characterization, laser-induced surface acoustic waves, electrode thin films, $\mathrm{V}_{2} \mathrm{O}_{5}$ 


\section{Introduction}

Lithium ion batteries are an integral part of today's energy storage landscape, but the constant miniaturization of electronic devices is currently challenging the technology to fit into reduced form factors. While smaller electronics have allowed for great advancement in many areas such as portable consumer electronics and biologically implantable devices [1], their energy requirements can often remain similar, if not greater than, before [2]. Thin film Li-ion batteries are capable of meeting the limited size requirements, but alternatives to the traditional graphite and lithium metal oxide electrode materials are necessary to maintain capacity and performance with diminished battery mass $[3,4]$. Nanostructured materials are of interest in this field because they can exhibit very high surface energies which enhance charge transfer kinetics and ion storage capacity [5-7]. Additionally, nanostructures, especially when coupled with porosity, can be more tolerant to the large physical deformations associated with Li-ion insertion and removal thus reducing the mechanical breakdown of the electrode and improving the battery life cycle $[8,9]$.

It is well known that the effects of the electrode volume change during each charge cycle, producing modest strains of $2 \%$ in $\mathrm{LiCoO}_{2}$ cathodes [10] and 7\% in $\mathrm{LiFePO}_{4}$ cathodes [11], contributes greatly to the loss of charge capacity over time [12]. Yet for such a mechanically rich process, there has been relatively little investigation correlating the breakdown to the mechanical properties of the electrode. While many studies have targeted and identified the main degradation mechanism, the proliferation of micro-cracks [13], comprehensive mechanical design criteria to mitigate such issues in new electrode materials have yet to be determined. With many other electrochemical considerations to contend with, it is easy to see why mechanical aspects are 
often overlooked, but with links between mechanics and rate capability [11] and the emergence of higher capacity materials with larger expected volume changes, nanomechanical characterization may become critical in understanding optimal electrode design.

The prevalence of nanoindentation in thin film mechanical analysis is especially valuable to the characterization of thin film electrodes. Indentation tests can quickly determine the elastic and plastic properties of a material with the elastic modulus being especially useful here. While electrode degradation is a complex problem with both electrochemical and mechanical considerations, the modulus can breathe insight into the flexibility and reliability of a material and thus aid in understanding how it may cope with lithiation induced strain. Several recent studies have successfully applied nanoindentation to electrode materials. Qu et al. reported on the elastic modulus, density, and fracture toughness of individual $\mathrm{LiCoO}_{2}$ grains and noted that the experimentally determined modulus of $174 \mathrm{GPa}$ was much lower than the previously predicted value of 315-516 GPa [14]. This suggests a more compliant structure than once thought. Ramdon and Bhushan reported on the elastic modulus, hardness, and wear properties of $\mathrm{LiFePO}_{4}$ cathodes before and after multiple charge cycles [15]. They found that the modulus did not change significantly through cycling, but that the hardness increased by almost $100 \%$ giving rise to a durable, yet brittle structure. Zhu et al. performed indentations on $\mathrm{RuO}_{2}$ anodes and found that the elastic modulus decreases by an order of magnitude after 50 cycles [16]. With such a limited quantity of studies, it is difficult to correlate the elastic modulus, or any other mechanical property, to battery performance over time therefore this area demands further investigation. 
The lack of nanomechanical studies could possibly be attributed to the difficulty of nanoindentation on thin film electrode materials. For consistent results that are free of indentation substrate effects, it is necessary to have films with low surface roughness and a thickness many times that of the penetration depth. This can be especially problematic to nanostructured or porous films since their engineered roughness may be too delicate for mechanical polishing and their thicknesses could be very small. While substrate effects from low thicknesses can be overcome by utilizing an alternative indentation analysis scheme [17], albeit with significant computational intensity, defeating roughness through deep indents may not always be viable. In this scenario, it may be more efficient to use a laser-induced surface acoustic wave (LiSAW) technique to determine the elastic modulus.

The LiSAW technique is valuable to thin and porous films because it requires only elastic deformations, it features non-contact measurements, and the mechanical property extraction naturally considers and isolates substrate coupling [18-20]. In the experiment, an Nd:YAG laser is used to excite Rayleigh waves that subsequently propagate across the layered system. Due to elastic mismatch between the film and substrate, frequency dispersion of the wave occurs. Since the relationship between mechanical properties and dispersion is well documented in wave mechanics [21], LiSAW can seek unknown parameters of the film or substrate by experimentally quantifying the dispersion. In this manner, if enough nonlinearity exists in the dispersion curve, the film thicknesses, Poisson's ratios, densities, and elastic moduli of the materials involved can be found. Typically, many of these parameters are known at the onset, thus only one or two parameters may require fitting. LiSAW can deliver these results on the same time-scale as typical indentation tests and with no substrate influence. Due to the micron scale of SAW 
wavelengths, the technique can work well on moderately rough films [22] and, depending on SAW detection scheme, with little or no surface preparation. While the LiSAW technique requires an area millimeters in scale for adequate wave dispersion with existing detection schemes, the creation of uniform blanket films of electronic materials is typically not a major challenge. Rather, it is the thickness of such films that creates the greatest characterization challenge and LiSAW directly addresses and excels at these geometries based on the inherently coupled film-substrate analysis procedure.

The limiting factor of many current Li-ion batteries lies in the low energy density of the cathode. Common cathode materials based on layered lithium metal oxides such as $\mathrm{LiCoO}_{2}$ and $\mathrm{LiFePO}_{4}$ have capacities of just $140 \mathrm{mAhg}^{-1}$ and $170 \mathrm{mAhg}^{-1}$ respectively [23]. Significant research has been completed on the oxides of other first row transition metals in order to achieve higher capacities. From these studies, vanadium oxides have emerged with great potential due to their wide range of available oxidation states, high reversibility, and favorable layered structures, all of which are conducive to the electrochemical lithiation process [24]. Specifically, $\mathrm{V}_{2} \mathrm{O}_{5}$, with a theoretical capacity of $450 \mathrm{mAhg}^{-1}$ [25], has been extensively studied and remains a leading candidate amongst alternative cathode materials. While $\mathrm{V}_{2} \mathrm{O}_{5}$ has been known for inadequate structural stability and slow electrochemical kinetics, nanostructured $\mathrm{V}_{2} \mathrm{O}_{5}$ forms have recently been shown to overcome these challenges by demonstrating excellent capacity, rate capability, and cyclability [26-28].

In this paper, the mechanical properties of a porous nanostructured $\mathrm{V}_{2} \mathrm{O}_{5}$ cathode film are investigated by nanoindentation and LiSAW. This particular film is especially attractive because 
it is synthesized by a simple and cost effective procedure via electrodeposition and exhibits excellent electrochemical properties. From a mechanical standpoint, the film's roughness and delicate nature, both properties common to nanostructured electrode materials and for which mechanical polishing is not viable, makes it an interesting specimen for testing the limits of traditional nanoindentation. The goal of this study is to perform LiSAW and discover whether the technique is reliable for such films. The results will then be compared to findings from nanoindentation with the intent of identifying the most efficient means for mechanical characterization of such media. It is hoped that the methodologies identified from this research will guide and fuel additional investigations in order to develop a further understanding of the relationship between electrode performance and its mechanical properties.

\section{Experimental Method}

\subsection{Electrodeposited $\mathrm{V}_{2} \mathrm{O}_{5}$ film synthesis}

For mechanical characterization, electrodeposited $\mathrm{V}_{2} \mathrm{O}_{5}$ films were prepared on single crystal silicon wafers with (100) orientation by the procedure reported in literature [25]. In brief, a vanadium pentoxide solution was formed by combining commercial $\mathrm{V}_{2} \mathrm{O}_{5}$ powder $(99.8 \%$ AlfaAESAR) with $\mathrm{H}_{2} \mathrm{O}_{2}$ (30 wt.\% in water, Sigma-Aldrich) and de-ionized water. The solution was stirred for 15 minutes and then sonicated for 15 minutes in water at constant temperature. Afterwards, the $\mathrm{V}_{2} \mathrm{O}_{5}$ concentration was diluted from $C_{V}=0.3 \mathrm{M}$ to $C_{V}=0.06 \mathrm{M}$ and the

resulting solution was sonicated for $1 \mathrm{~h}$. A final dilution, this time to $C_{V}=0.0075 \mathrm{M}$, completed the $\mathrm{V}_{2} \mathrm{O}_{5}$ solution and prepared it for use in electrodeposition. 
Prior to electrodeposition, the Si substrates were sputter coated with less than $5 \mathrm{~nm}$ thick gold palladium to enhance conductivity. They were then lowered into the $\mathrm{V}_{2} \mathrm{O}_{5}$ solution parallel to a platinum plate counter electrode. Deposition was carried out at $-2.4 \mathrm{~V}$ for $1 \mathrm{~h}$ with a separation distance between the electrodes of $\sim 15 \mathrm{~mm}$. Upon completion, the films were dried in air at 70 ${ }^{\circ} \mathrm{C}$ for $1 \mathrm{~h}$ and then annealed in air at $450{ }^{\circ} \mathrm{C}$ for $3 \mathrm{~h}$. To obtain free standing films for density measurements, the procedure was carried out on nickel foil substrates (99.9\% Sigma-Aldrich) instead of silicon. After electrodeposition, the undried gel films were carefully detached from the foil and bridged across a $20 \mathrm{~mm}$ gap on wax paper. The films were dried and cut free from the wax paper to isolate their spanning areas prior to annealing. Drying and annealing conditions remained unchanged from before. All film thicknesses were measured by profilometry (Dektak, Tucson, AZ).

\subsection{Electrochemical Analysis of $\mathrm{V}_{2} \mathrm{O}_{5}$ Films}

For electrochemical analysis, $\mathrm{V}_{2} \mathrm{O}_{5}$ thin film electrodes were deposited using the same procedure described above but with a deposition time of 30 min on FTO glass and annealing temperature of $500{ }^{\circ} \mathrm{C}$. The slightly higher temperature was chosen to assure the complete removal of the crystalline water in the $\mathrm{V}_{2} \mathrm{O}_{5}$ interlayers since non-aqueous solvent is used in the electrochemical testing. However, the $50{ }^{\circ} \mathrm{C}$ difference in annealing temperature is not expected to produce any discernable difference in the microstructure and mechanical properties of the film. The electrodes were tested using a standard three-electrode configuration with $1 \mathrm{M} \mathrm{LiClO}_{4}(98 \%$, Sigma Aldrich) in propylene carbonate (99.7\%, Sigma Aldrich) as electrolyte, a Pt plate as counter electrode, and $\mathrm{Ag} / \mathrm{Ag}^{+}$as reference electrode (CH Instruments). Both Cyclic voltammetry $(\mathrm{CV})$ and chronopotentiometry $(\mathrm{CP})$ tests were performed using an electrochemical analyzer (CH Instruments, Model 605B). CV was conducted in the voltage range of 0.6 to $-1.8 \mathrm{~V}$ 
(vs. $\mathrm{Ag} / \mathrm{Ag}^{+}$) at a scan rate of $5.0 \mathrm{mV} \mathrm{s}{ }^{-1}$. CP was also performed in the voltage range of 0.6 to $1.8 \mathrm{~V}$ (vs. $\mathrm{Ag} / \mathrm{Ag}^{+}$) assuming a $1 \mathrm{C}$ current density of $298 \mathrm{~mA} \mathrm{~g}^{-1}$ corresponding to the total insertion of two lithium ions per $\left[\mathrm{V}_{2} \mathrm{O}_{5}\right]$ formula unit.

\subsection{Mechanical Characterization by Nanoindentation}

Nanoindentation measurements were performed by a Hysitron UBi1 nanomechanical test instrument (Hysitron, Minneapolis, MN) with a diamond Berkovich tip $\left(142.3^{\circ}\right.$ total included angle). All indentations followed a trapezoidal loading curve featuring a $10 \mathrm{~s}$ ramp loading segment, a $5 \mathrm{~s}$ hold at maximum load, and a $10 \mathrm{~s}$ unloading period. To decrease the likelihood of substrate influence, maximum indentation depths were contained to $10 \%$ of the film thickness when possible. Mechanical properties were derived from indentation loading curves by the common Oliver and Pharr method [29].

\subsection{LiSAW Experimental Setup}

LiSAW measurements were performed by the apparatus shown in Fig. 1. The setup is highlighted by a 5 ns pulse width Nd:YAG laser (New Wave Research, Fremont, CA) operating at $1064 \mathrm{~nm}$ for SAW generation and a continuous wave argon-ion laser (Coherent, Santa Clara, CA) operating at $514.5 \mathrm{~nm}$ for SAW detection. For planar SAW generation, the circular Nd:YAG beam was focused by a cylindrical lens to a line source on the specimen surface with a width of $\sim 8 \mu \mathrm{m}$. During SAW detection, out-of-plane surface displacements were measured by the argon-ion laser in a Michelson interferometer configuration. Displacement for this type of interferometer is obtained through laser interference as the light is split into two paths by the beam splitter, one to the sample and one to a reference mirror, then later recombined. To 
maximize frequency detection, the beam was expanded prior to focusing by two identical shortfocal distance lenses to achieve a circular spot size of $<10 \mu \mathrm{m}$ on the sample and reference mirror. The interference signal was detected by a $1.2 \mathrm{GHz}$ photo detector (EOT, Traverse City, MI) with a $300 \mathrm{ps}$ rise/fall time and then digitized by a $5 \mathrm{GHz}$ oscilloscope sampling at a rate of 20 gigasamples/sec (LeCroy, Chestnut Ridge, NY).

In a Michelson interferometer, the voltage signal recorded by the oscilloscope will oscillate as the sample moves in time. The signal is directly related to sample displacement such that a sample movement of $\lambda / 4$, where $\lambda$ is the laser wavelength, represents half of a fringe (i.e. maximum destructive interference to maximum constructive interference). Since SAW displacements were much lower than $\lambda / 4$ for our setup, the detection was considered sub-fringe. In this regime, mid-fringe measurements were necessary since in that region, the displacement sensitivity is considerably higher than in other regions of the fringe. This is because of the sinusoidal nature of fringe intensity with respect to surface displacement. Near the peaks and troughs of fringes, sample displacement produces less change in light intensity since the slope is nearer to zero. Conversely, between the peak and trough (mid-fringe), the slope is highest thus generating the largest change in light intensity per unit of displacement. 


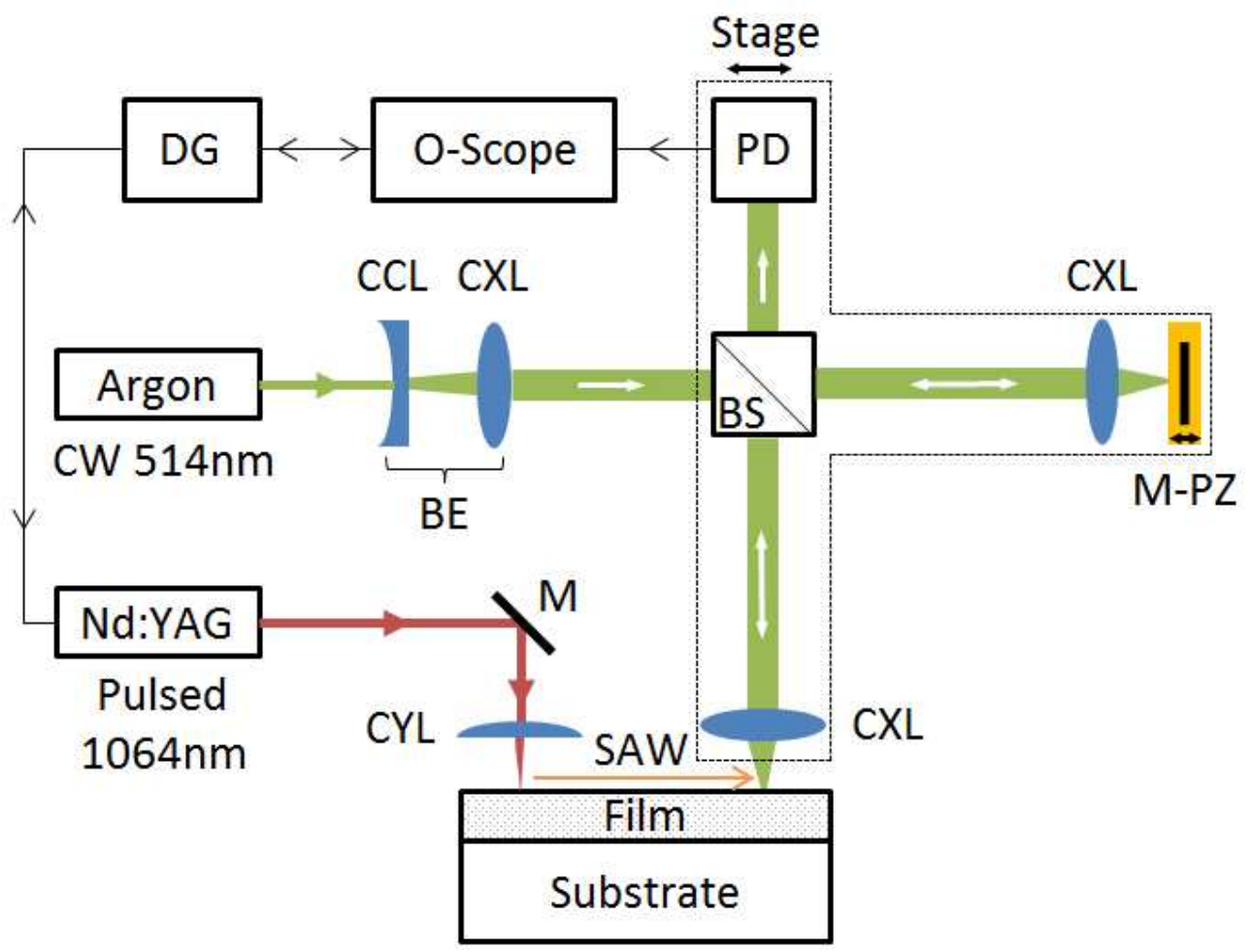

Fig. 1 LiSAW experimental setup. Abbreviations: DG: delay generator, O-scope: oscilloscope, PD: photo detector, CXL: convex lens, CCL: concave lens, BE: beam expander, BS: beam splitter, M-PZ: mirror mounted on piezoelectric stage, CYL: cylindrical lens.

SAW events were synchronized to the middle linear fringe region with a technique similar to that reported by the authors previously [30]. In brief, a piezoelectric stage was mounted on the reference mirror and to set to oscillate. This produced an interference pattern with consistent and repeatable temporal wavelengths (Fig. 2) that could be tuned to the timescale of the generation laser firing operation (several hundred microseconds) by adjusting the piezoelectric vibration frequency. An oscilloscope monitoring signal rise and fall times could then detect selected wavelengths and use them to trigger and time the laser output to the desired linear fringe region. This synchronization technique relies on the ability to oscillate the mirror fast enough to overcome background noise, yet slow enough so as to not impede on the SAW timescale. Typically, a frequency of $100-200 \mathrm{~Hz}$ with a mirror movement of several microns was sufficient. 


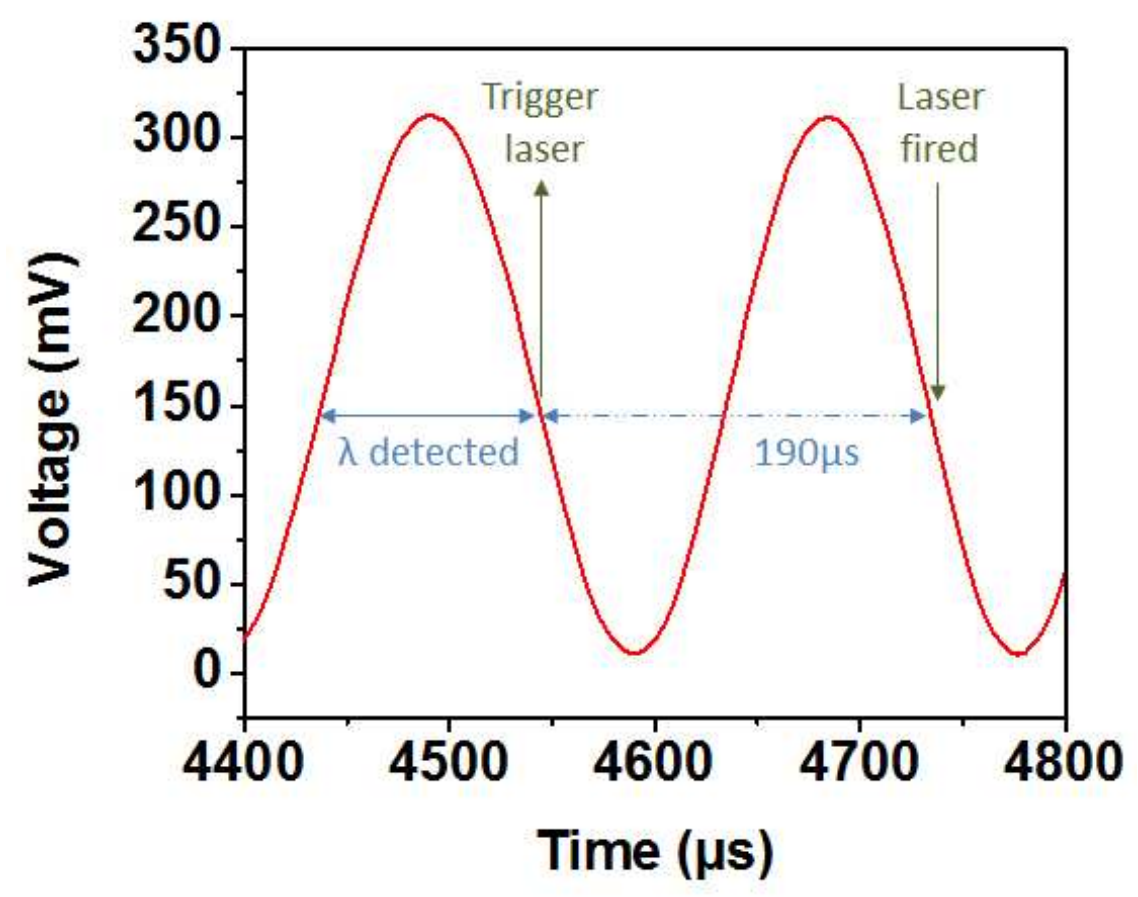

Fig. 2 Schematic of synchronization scheme that allows for mid-fringe laser firing for optimal displacement sensitivity.

\subsection{SAW Signal Processing and Mechanical Property Extraction}

The detected SAW signals were inherently noisy with contamination from background vibrations and high frequency electronics. To suppress this noise, multiple signals were averaged together and a Fourier filter with a passing band of $15-300 \mathrm{MHz}$ was used. To isolate the SAW event, a Tukey window function was employed with $\beta=0.25$. Since SAW dispersion presents as a physical waveform change during propagation, two SAW signals, with known spacing from one another, are required for dispersion analysis. After obtaining the signals on each sample, dispersion was calculated through Eq. 1:

$$
c(f)=\frac{2 \pi f\left(d_{2}-d_{1}\right)}{\Phi_{2}(f)-\Phi_{11}(f)}
$$


where $c$ is phase velocity, $f$ is frequency, $d_{2}-d_{1}$ is the spacing between detection sites, and $\Phi$ is the phase spectrum for each signal. This produced the characteristic dispersion plot of phase velocity versus frequency.

To extract mechanical properties, the experimental dispersion curves were fit with theoretically generated curves where the elastic modulus or density was the fitting parameter. Since only linear dispersion was seen, only one parameter could be fit. The derivation of theoretical dispersion curves with assumed substrate and film mechanical properties followed the same procedure as detailed in literature [21]. In brief, particle displacement was modeled with a generalized wave equation (Eq. 2) and coupled to material properties through the governing equation of motion (Eq. 3).

$$
\begin{aligned}
& u_{j}=\alpha_{j} \exp \left(i k b x_{3}\right) \exp \left[i k\left(x_{1}-v t\right)\right] \\
& \rho \frac{\partial^{2} u_{j}}{\partial t^{2}}=c_{i j k l} \frac{\partial^{2} u_{k}}{\partial x_{i} \partial x_{l}} \quad(i, j, k, l=1,2,3)
\end{aligned}
$$

In Eqs. 2 and 3, $u_{j}$ is displacement, $\alpha_{j}$ is relative amplitude, $k$ is the wave vector, $b$ is a complex parameter that governs wave decay, $v$ is wave velocity, $t$ is time, and $c_{i j k l}$ is the stiffness tensor. By inserting Eq. 2 into Eq. 3, a system of 3 equations results for each material that contains an eigenvalue problem that can be solved for $b$ with assumed wave velocity and mechanical constants. With $b$ 's known for all materials involved, boundary conditions are considered that are comprised of stress and displacement continuity at the interface and zero stress at the free surface. The boundary conditions form a set of equations that can be solved together to seek the wave vector, $k$, which houses frequency information. The analysis is repeated for many phase velocities to form a theoretical dispersion plot of phase velocity versus frequency for assumed 
mechanical properties. In the cases where a secondary reflective layer was used for facilitating optical detection, the derivation followed a similar procedure [31] to account for the second top film. The latter derivation applies to this work.

\section{Results and Discussion}

\subsection{Structure Confirmation and Electrochemical Performance}

Two samples were synthesized for LiSAW testing with each having dimensions of $\sim 25 \times 25 \mathrm{~mm}$, while the sample synthesized for electrochemical analysis had dimensions of $\sim 10 \times 10 \mathrm{~mm}$. The electrodeposited films were characterized by X-ray diffraction to confirm the presence of $\mathrm{V}_{2} \mathrm{O}_{5}$. The resulting spectrum, shown in Fig. 3, clearly displays the prominent (001) and (101) peaks of orthorhombic $\mathrm{V}_{2} \mathrm{O}_{5}$ thus confirming the oxide structure. Fig. 4 shows the rough nanostructured surface as imaged by a scanning electron microscope. The film thicknesses for mechanical characterization were measured to be $1.7 \mu \mathrm{m}$ and the film thickness for the electrochemical analysis is $\sim 800 \mathrm{~nm}$. The two sets of films are structurally identical. It was the intent of the authors to create thicker films for the mechanical characterization in order to isolate the challenge of nanostructured roughness from the substrate effect during latter indentation tests. 


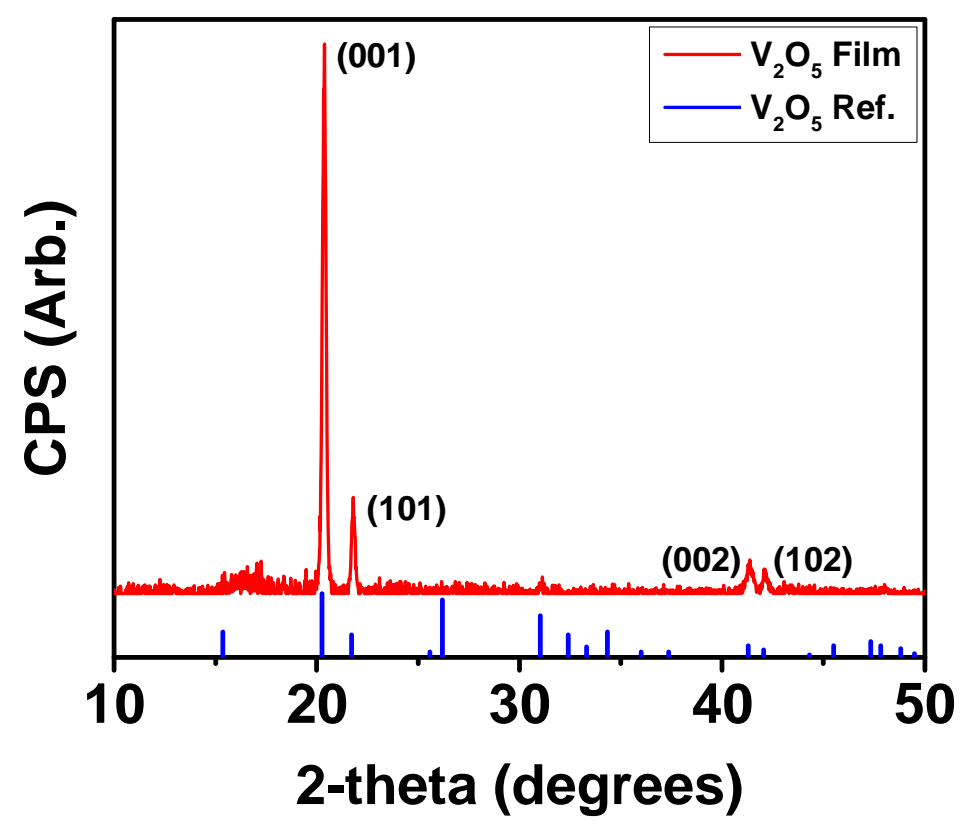

Fig. 3 X-ray diffraction spectrum of the nanostructured $\mathrm{V}_{2} \mathrm{O}_{5}$ film compared to a reference spectrum [32].

Nitrogen adsorption isotherms were collected in order to evaluate the surface area and pore characteristics. The Barrett-Joyner-Halenda $(\mathrm{BJH})$ pore size distributions obtained suggest that the sample contains broadly distributed pores, the majority of which are smaller than $15 \mathrm{~nm}$, with an average pore size of $3.19 \mathrm{~nm}$; the BJH pore volume was $0.054 \mathrm{~cm}^{3} \mathrm{~g}^{-1}$ while the BrunauerEmmett-Teller (BET) derived specific surface area was $32.58 \mathrm{~m}^{2} \mathrm{~g}^{-1}$. The nanoscale pore size does not pose challenges to characterization by LiSAW and nanoindentation since both techniques involve microscale interactions, such as the physical wavelengths in LiSAW and tip radii in nanoindentation, which are both multiple orders of magnitude larger than the pore diameter. 


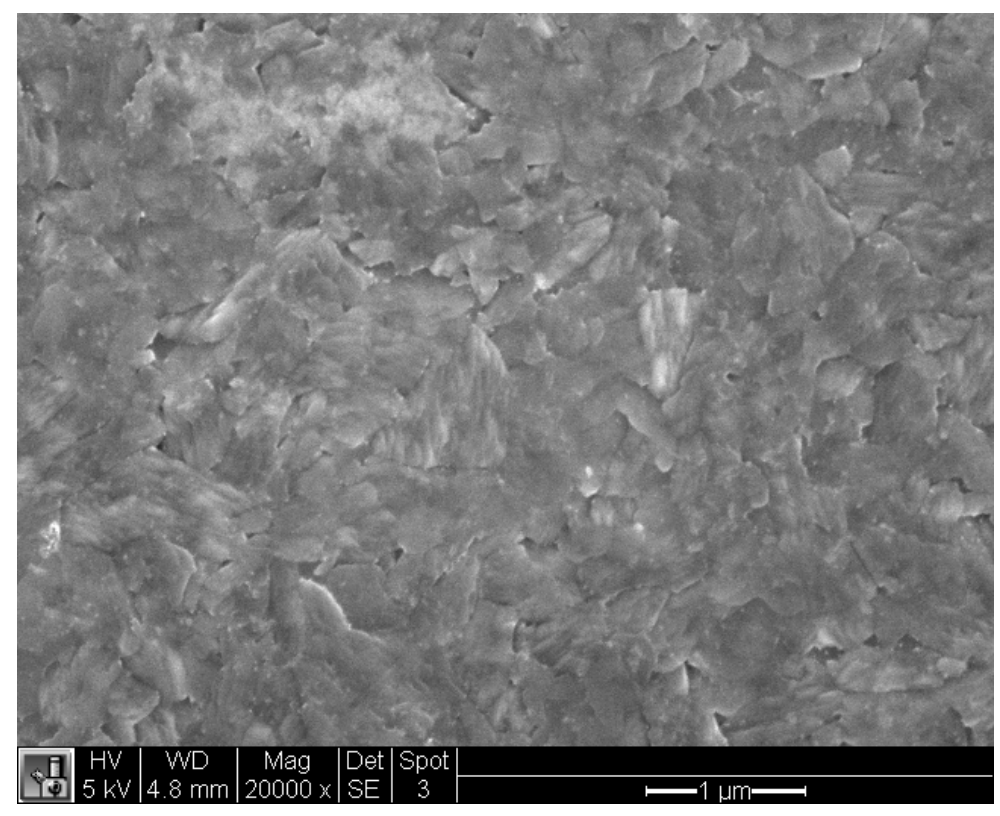

Fig. 4 Image of the $\mathrm{V}_{2} \mathrm{O}_{5}$ surface taken by a scanning electron microscope.

To confirm the electrochemical performance, $\mathrm{CV}$ was performed to elucidate the redox processes at play for the $\mathrm{V}_{2} \mathrm{O}_{5}$ thin film electrodes, the first and eighth cycles of which are shown in Fig. 5(a). There are at least three clearly observable reduction and oxidation peaks for the initial CV scan, but these peaks become attenuated and broaden with further cycling; such findings are typically attributed to a loss of electro-active material crystallinity due to the lithium insertion/extraction process [33]. Regardless, the reduction peaks that accompany lithiation at $0.20,-0.40$, and $-1.65 \mathrm{~V}$ vs. $\mathrm{Ag} / \mathrm{Ag}^{+}$can be ascribed to several well documented $\mathrm{V}_{2} \mathrm{O}_{5}$ phase transitions, namely $\alpha / \varepsilon, \varepsilon / \delta$, and $\delta / \gamma$, respectively. Conversely, the oxidation peaks at $-0.7,-0.18$, and $-0.008 \mathrm{~V}$ vs. $\mathrm{Ag} / \mathrm{Ag}^{+}$correspond to the $\gamma / \delta, \delta / \varepsilon$, and $\varepsilon / \alpha$ phase transitions, respectively [3436]. Comparison of these two cyclic voltammograms also reveals a slight drop in the overall current with cycling that can be ascribed to solid electrolyte interphase (SEI) formation. The formation of this organic and inorganic dual layered product, formed through electrolyte decomposition, effectively sequesters and intercepts lithium, leading to the subsequent drop in 
current [37]. The potential difference between the oxidation and reduction peaks of each redox couple, which is a relative measure of the reversibility of the redox reaction, also diminishes with cycling. These considerations ultimately prove that the electrode is becoming stable in the later stages of cycling.

Fig. 5(b) shows the discharge capacity at a current density of $200 \mathrm{~mA} \mathrm{~g}^{-1}(0.67 \mathrm{C})$ for the first 20 cycles, followed by 120 cycles of discharge/charge tests at various current densities. The initial discharge capacity is $410 \mathrm{~mA} \mathrm{~h} \mathrm{~g}^{-1}$, which surpasses the theoretical capacity corresponding to the insertion of two $\mathrm{Li}^{+}$per $\mathrm{V}_{2} \mathrm{O}_{5}$ formula unit but is in actuality an inflated value because of SEI layer formation. Following this, the second discharge capacity dropped to $352 \mathrm{~mA} \mathrm{~h} \mathrm{~g}{ }^{-1}$, and then stayed at $320 \mathrm{~mA} \mathrm{~h} \mathrm{~g}^{-1}$ up to the 20th cycle. The coulombic efficiency for the initial and twentieth cycles is $83.2 \%$ and $98.9 \%$, respectively. The initial discharge capacity at current densities of $0.67,1.5,5,15$, and $35 \mathrm{C}$ were $279,220,164$, and $126 \mathrm{~mA} \mathrm{~h} \mathrm{~g}^{-1}$, respectively. After more than 130 cycles tested at different current densities, the current rate was returned to $1.5 \mathrm{C}$ and the discharge capacity was still as high as $256 \mathrm{~mA} \mathrm{~h} \mathrm{~g}^{-1}$. The specific energy densities were calculated to be $700 \mathrm{~W} \mathrm{~h} \mathrm{~kg}^{1}$ for the twenty-first cycle and $670 \mathrm{~W} \mathrm{~h} \mathrm{~kg}^{-1}$ for the 150 th cycle when discharged at $450 \mathrm{~mA} \mathrm{~g}^{-1}(1.5 \mathrm{C})$.

The high electrochemical performance demonstrated here could be ascribed to the nanometric scale of the $\mathrm{V}_{2} \mathrm{O}_{5}$ thin film electrodes which can provide a shorter diffusion path for Li-ion intercalation/deintercalation, and a higher surface area that offers more accessible intercalation sites which favors electrolyte penetration and interfacial reactions. As evidenced by the LiSAW results later, the externally applied electrical field during deposition assists adhering the film to 
the conductive substrate which facilitates Li-ion diffusion and enables excellent energy and power density; the improvement in mechanical integrity is especially apparent at high current densities.
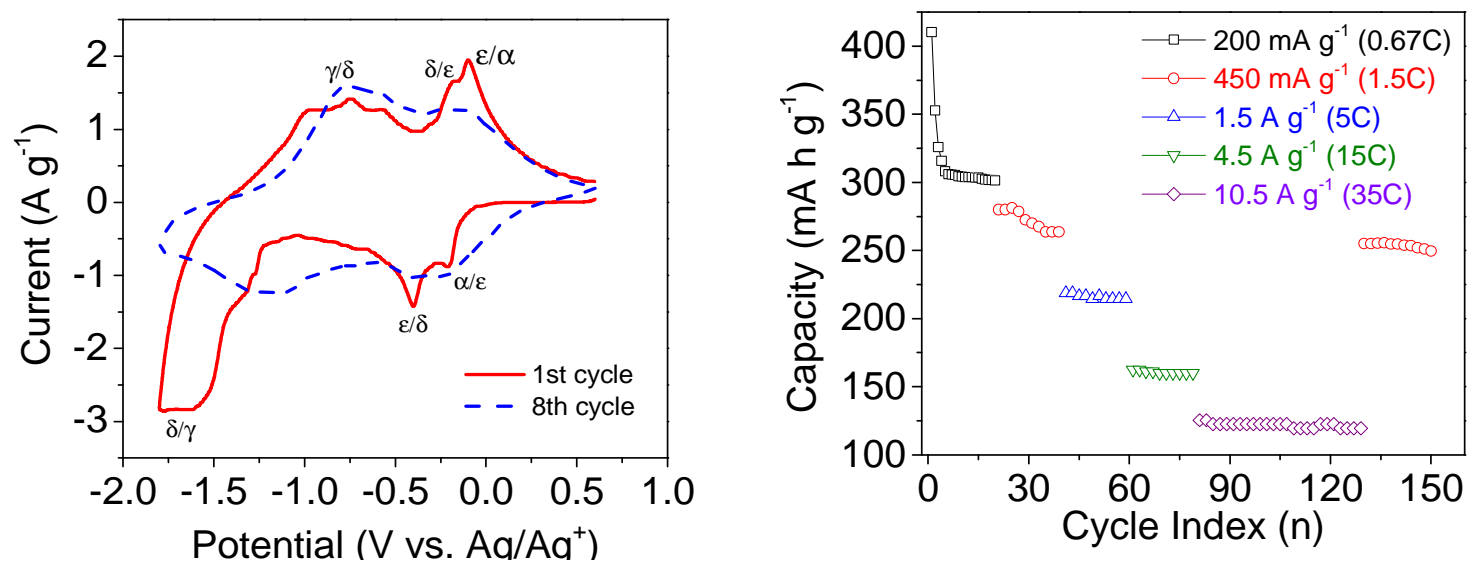

Fig. 5 Electrochemical performance of the $500^{\circ} \mathrm{C}$ annealed $\mathrm{V}_{2} \mathrm{O}_{5}$ thin film electrodes: (a) cyclic voltammogram at a scan rate of $5 \mathrm{mV} \mathrm{s}^{-1}$ for the initial and eighth cycles, (b) rate capability and cyclic stability at various current densities.

\subsection{Mechanical Properties by LiSAW}

When performing the electrodeposition, low resistivity silicon wafers were used for substrates. Heavy doping, with impurity concentrations on the order of $10^{19} \mathrm{~cm}^{-3}$, was necessary to achieve resistivities $<0.002 \Omega$-cm and this has been shown to modify the elastic constants of silicon [38]. While the variations in elastic parameters are expected to be $\sim 1 \%$ for the doping levels here, they can still have a significant effect on Rayleigh wave speed $\left(c_{r}\right)$. To account for the change, LiSAW was performed on bare silicon wafers prior to film deposition. The results were used to fit the $c_{11}$ stiffness tensor for subsequent analysis in theoretical curve determination. The doped wafers were found to have $c_{r}=4896 \mathrm{~m} / \mathrm{s}$ in the [100] direction which is a bit lower than the results for undoped wafers $\left(c_{r}=4917 \mathrm{~m} / \mathrm{s}\right)$. This corresponded to $-1 \%$ decrease in $c_{11}$ 
lowering it from 165.7 GPa to $163.8 \mathrm{GPa}$. This value was used for all theoretical calculations to follow. The other silicon constants were assumed to be their theoretical values of $c_{12}=$ $63.9 \mathrm{GPa}, c_{44}=79.6 \mathrm{GPa}$, and $\rho=2330 \mathrm{~kg} / \mathrm{m}^{3}$.

To make the $\mathrm{V}_{2} \mathrm{O}_{5}$ surface more reflective for optical detection methods, a thin layer of aluminum was deposited on top of the $\mathrm{V}_{2} \mathrm{O}_{5}$ by magnetron sputtering (AJA International, Scituate, MA) as a secondary top film. The elastic modulus of the sputtered aluminum films, a key parameter for theoretical fitting, was confirmed by performing LiSAW and nanoindentation on separate samples without $\mathrm{V}_{2} \mathrm{O}_{5}$. Both techniques measured a modulus of $70 \mathrm{GPa}$ which is in agreement with literature [39]. The other properties of aluminum used for theoretical dispersion curves were $\rho=2700 \mathrm{~kg} / \mathrm{m}^{3}$ and $v=0.33$.

The first $\mathrm{V}_{2} \mathrm{O}_{5}$ film tested with LiSAW received a $100 \mathrm{~nm}$ aluminum top layer. SAWs were generated in the [100] direction of the silicon substrate and detected after traveling various distances ranging between $10 \mathrm{~mm}$ and $15 \mathrm{~mm}$ from the irradiation site. SAW waveforms obtained at $10 \mathrm{~mm}$ and $15 \mathrm{~mm}$ are shown in Fig. 6. Dispersion of the signals is evident from the waveform elongation on the $15 \mathrm{~mm}$ signal, especially visible at low frequencies. Spectral analysis showed a frequency response between $15-60 \mathrm{MHz}$ in both signals. The upper bound of the detected frequencies is quite low considering that the detection spot size of $<10 \mu \mathrm{m}$ is sufficient to identify frequencies well in excess of $100 \mathrm{MHz}$. However, the nanostructured roughness was a likely cause of high frequency attenuation. It should be noted that the penetration depth of SAWs is on the order of one wavelength therefore a $50 \mathrm{MHz}$ wave 
propagating on silicon has an influence of almost $100 \mu \mathrm{m}$. The film region represents only a small fraction of this influence, yet significant detectable dispersion occurs even at low frequencies. Thus, while a large bandwidth is desirable, it is not always necessary, as is the case in this sample where there was more than adequate bandwidth for dispersion analysis. This reasoning is why LiSAW is an attractive and effective technique for very thin films of delicate nature.

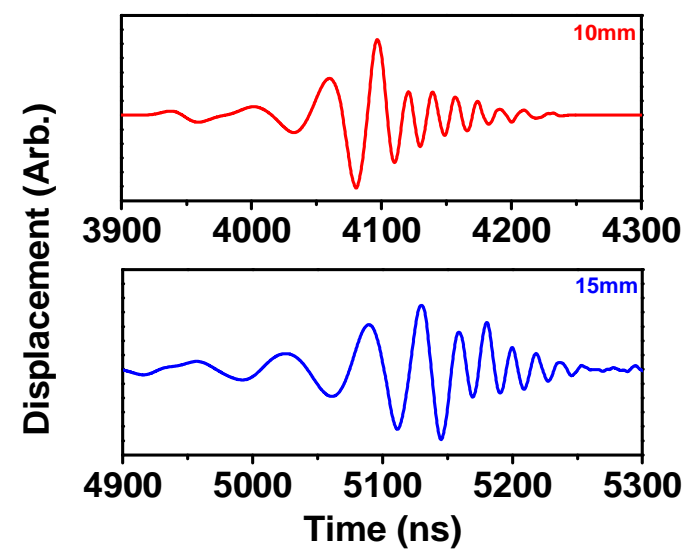

Fig. 6 SAW signals recorded on the first $\mathrm{V}_{2} \mathrm{O}_{5}$ sample at a distance of $10 \mathrm{~mm}$ and $15 \mathrm{~mm}$ from the source. To aid in shot to shot SAW alignment, the $x$ timescale is offset by $2 \mu \mathrm{s}$ such that sample irradiation occurs at $\sim 2000 \mathrm{~ns}$.

Experimental dispersion curves were calculated for the signals in Fig. 6 and the elastic modulus was extracted through fitting of theoretical curves. Four film parameters are necessary for fitting: Poisson's ratio, film thickness, density, and elastic modulus. Since we have linear dispersion, three of the four parameters must be known in advance. Fortunately, the Poisson's ratio ( 0.3 was used) has a negligible influence on dispersion [31] and the thickness $(1.7 \mu \mathrm{m})$ can be measured to a high degree of certainty. Density can typically be determined by methodologies involving Archimedes principle such as the case in this study where a pycnometer was utilized. The measured density was $2.28 \pm 0.11 \mathrm{~g} / \mathrm{cm}^{3}$. As mentioned previously, the BJH pore volume was 
measured to be $0.054 \mathrm{~cm}^{3} / \mathrm{g}$ which suggests a film density of $2.84 \mathrm{~g} / \mathrm{cm}^{3}$. While this is considerably higher than the value obtained by pycnometry, it assumed that all internal sample pores were intruded on in the powder sample. Any discrepancy would lower the density further. It should be noted that both measurements are considerably less dense than an ideally packed $\mathrm{V}_{2} \mathrm{O}_{5}$ structure which has a density of $3.36 \mathrm{~g} / \mathrm{cm}^{3}$ thus confirming substantial film porosity. The value given by pycnometry was utilized for analysis since it provides the best estimate of the bulk macroscale density for which LiSAW wave propagation characteristics are derived.

With all of the substrate and film parameters accounted for, the experimental dispersion curve was fit to a modulus of $53 \pm 4 \mathrm{GPa}$. The error bars are based on the uncertainty in density measurement since this property dominates over other sources of error. The error was resolved by creating theoretical dispersion curves with the bounds given by the density measurement at a constant modulus of $53 \mathrm{GPa}$. These produced upper and lower bounding dispersion curves for which the elastic modulus was then fitted to thereby producing upper and lower modulus bounds. Fundamentally, the resolution in separation distance, spectral bandwidth of the probe laser, and time resolution of the detection electronics also contributes to error, but all of these combined would produce $<1 \mathrm{~m} / \mathrm{s}$ of error in phase velocity for a $100 \mathrm{MHz}$ signal. This is substantially smaller than the $10+\mathrm{m} / \mathrm{s}$ in error that the density uncertainty provides. Furthermore, curve fitting error was low and did not contribute significantly to error since the experimental curves were very linear and thus correlated well with the fit $\left(R^{2}=0.968\right)$. 


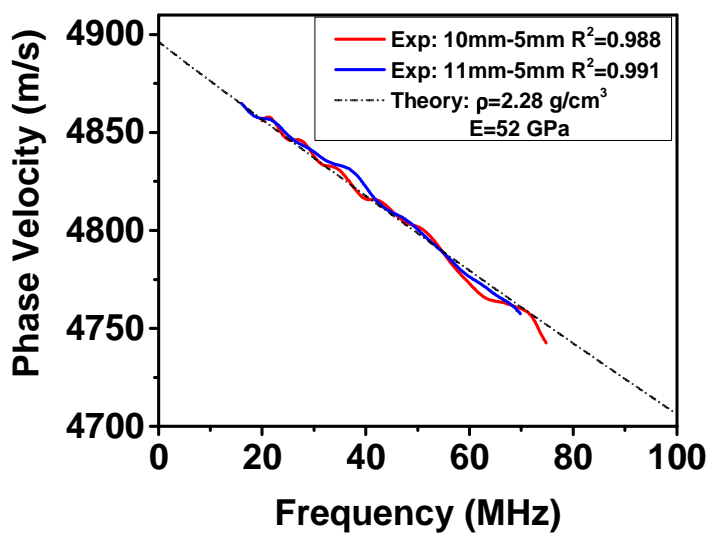

Fig. 7 Dispersion curves and theoretical fit for the second $\mathrm{V}_{2} \mathrm{O}_{5}$ sample.

A second $\mathrm{V}_{2} \mathrm{O}_{5}$ film, with identical specifications as the first film, was tested to demonstrate measurement repeatability. On this sample, a $200 \mathrm{~nm}$ aluminum reflective layer was sputtered prior to testing. The SAWs were detected at smaller propagation distances, $5-11 \mathrm{~mm}$, in an effort to capture higher frequencies before they attenuated. This resulted in a slight increase to the frequency limit with a new range of $15-75 \mathrm{MHz}$. The experimental dispersion curves generated from combinations of signals detected at $5 \mathrm{~mm}, 10 \mathrm{~mm}$, and $11 \mathrm{~mm}$ are shown in Fig. 7. Theoretical curves were generated and the best fit curve (Fig. 7) revealed a modulus of $52 \mathrm{GPa}$ which was in excellent agreement to the first sample.

\subsection{Mechanical Properties by Nanoindentation}

Nanoindentation was performed on the first of the two LiSAW samples described above. The test preceded the LiSAW study and was conducted before the reflective aluminum layer was deposited. The in-situ scanning probe microscopy (SPM) capability of the indentation system allowed for surface analysis prior to indentation. An SPM topography image is shown in Fig. 8. The roughness of the film is visibly evident and the measured RMS roughness resulting from a 
$10 \times 10 \mu \mathrm{m}$ area was $40 \mathrm{~nm}$. Manual polishing was attempted with $0.05 \mu \mathrm{m}$ alumina powder on a low nap fine polishing cloth (Ted Pella, Redding, CA), but the film delaminated and detached too easily. Thus while the film/substrate interface integrity is good enough to survive LiSAW, it could not survive the polish process even with just the sample holder weight of approximately $100 \mathrm{~g}$ applied. As a result, nanoindentation were performed on the as-synthesized state without polishing.

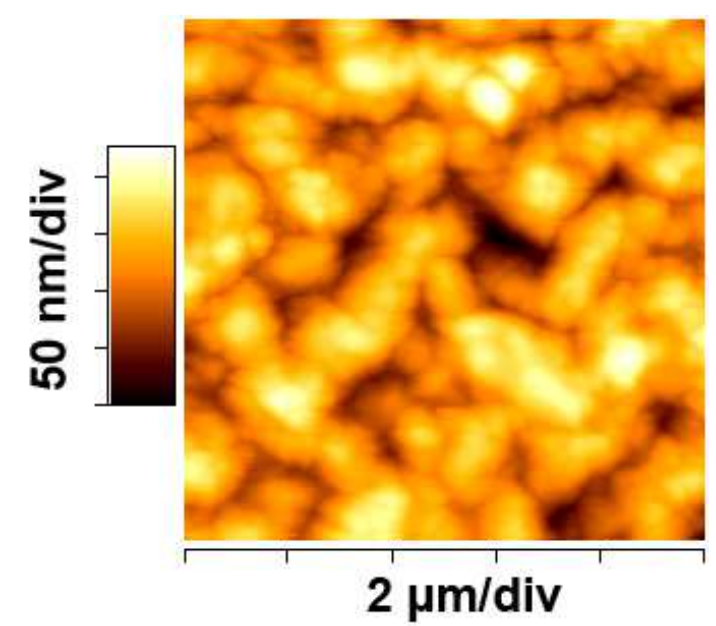

(a)

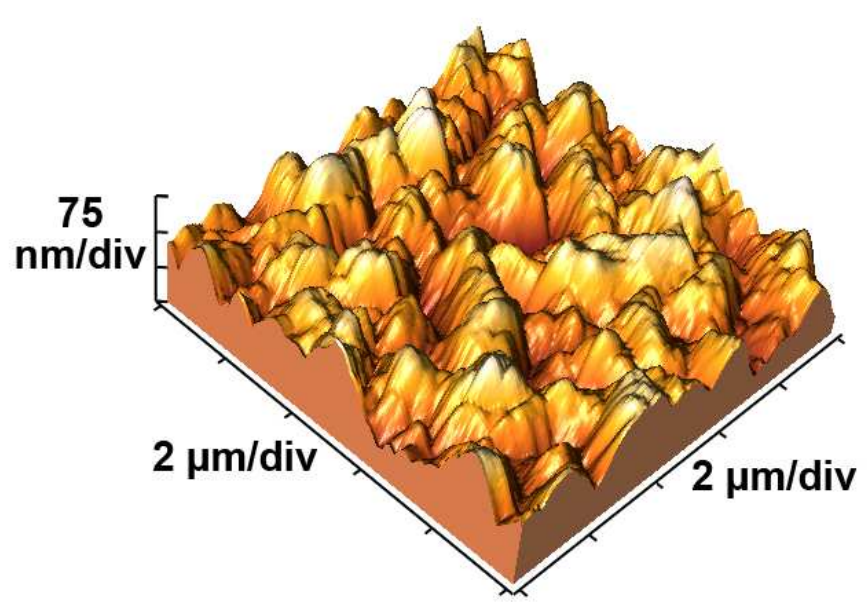

(b)

Fig. $810 \times 10 \mu \mathrm{m}$ SPM image of the $\mathrm{V}_{2} \mathrm{O}_{5}$ surface: (a) 2D topography, (b) 3D profile.

Initially, nanoindentation proceeded to depths of below $80 \mathrm{~nm}$ in order to simulate characterization of the thickest film for which electrochemical data was reported on previously. This corresponds to $\sim 10 \%$ (in order to minimize substrate effects) of the $800 \mathrm{~nm}$ thick film used for the electrochemical analysis in this study. For thin film electrodes, this $800 \mathrm{~nm}$ thickness is still quite large since most are considerably thinner (i.e. $3 \mathrm{~min}, 125 \mathrm{~nm}$ film) in order to minimize Li-ion diffusion time and thus maximize power capability [40]. Indentations were performed ranging from $13-74 \mathrm{~nm}$ in maximum depth and the calculated film modulus was $50 \pm 10 \mathrm{GPa}$. The error bars represent the $1 \sigma$ value resulting from a total of 74 measurements across many 
areas on the sample surface. It is apparent that the roughness is a likely culprit for the high standard deviation since many of the indentations were comparable in size to surface asperities. Previous measurements on a smooth film with the same tip, similar indentation depths, and a similar film moduli produced a $1 \sigma$ error of $<1.5 \mathrm{GPa}[41]$.

It should be noted that the modulus extracted from indentation loading curves is known as the reduced modulus (or indentation modulus), $E_{r}$, and its value represents a combination of film and indenter tip properties. Eq. 4 can be used to isolate the film modulus, $E_{f}$, requiring the tip properties (modulus $E_{i}=1140 \mathrm{GPa}$ and Poisson's ratio $v_{i}=0.07$ for the diamond Berkovich tip) and the film Poisson's ratio $\left(v_{f}\right)$, but unlike LiSAW, the extraction is quite sensitive to the Poisson's ratio.

$$
\frac{1}{E_{r}}=\frac{\left(1-v_{i}^{2}\right)}{E_{i}}+\frac{\left(1-v_{f}^{2}\right)}{E_{f}}
$$

A Poisson's ratio of 0.3 (identical to LiSAW fitting) was used to obtain the $50 \mathrm{GPa}$ value reported above, but considering a range of $v=0.2-0.35$, the modulus can differ by as much as 47.9 - 52.4 GPa. Thus, uncertainty in the Poisson's ratio can affect the nanoindentation measurement error in addition to the normal site to site error; in this case, the additional error was $4-5 \%$. A similar argument can be made about the LiSAW technique which requires and is sensitive to the film density. However, density is typically less challenging to procure by direct means and the site to site error is lower for LiSAW since the measurement naturally averages over the SAW propagation distance. 
A second round of indentation was performed to larger depths in order to explore whether the roughness could be overcome. The maximum depth was extended to $170 \mathrm{~nm}$ representing $10 \%$ of the film thickness. The indentations revealed a modulus of $51 \pm 9 \mathrm{GPa}$, a slight increase compared to the previous tests with an improvement on the error. The increase is well within the error bars of the previous test, but could be explained by possible collapse of the porous network at shallow indentation depths prior to densification at larger depths [42]. Different from our previous work on a thin porous zeolite film [30], no substrate effects were witnessed in the current study since the film was sufficiently thick. Overall, the nanoindentation data produced results similar to the LiSAW tests, but with substantially higher measurement error. Considering the film roughness of $40 \mathrm{~nm}$, even the deeper indentations could easily be affected by the surface features. This highlights one of the advantages of the LiSAW technique by demonstrating accurate analysis on a rough nanostructured film.

\section{Conclusion}

From the studies performed, it is clear that both LiSAW and nanoindentation are valuable techniques in the field of thin film mechanical electrode materials characterization. They were both able to measure the elastic modulus of the porous nanostructured $\mathrm{V}_{2} \mathrm{O}_{5}$ electrode, but LiSAW was more accurate on the rough film. Two LiSAW samples were tested resulting in an elastic modulus of $53 \pm 4 \mathrm{GPa}$ for the first sample and $52 \pm 4 \mathrm{GPa}$ for the second. Both samples exhibited very linear dispersion curves which correlated well to the theoretical fit, thus the major contribution to error came from uncertainty in the film density. Nanoindentation produced a modulus of $50 \pm 10 \mathrm{GPa}$ at shallow indentation depths and $51 \pm 9 \mathrm{GPa}$ at depths up to $170 \mathrm{~nm}$, or $10 \%$ of the film thickness. Roughness had a pronounced effect as evident by the large standard 
deviations. Uncertainty in the film Poisson's ratio, which is necessary to extract elastic modulus from indentation loading curves, can contribute an additional 4-5\% error for this film if $v=$ $0.2-0.35(v=0.3$ was used) are considered. The slightly lower results from nanoindentation could be the result of indentation induced pore collapse at low loading. However it is noted that the results are all within the error bars of one another, although the uncertainty of nanoindentation measurements was considerably larger. Overall, while the results extracted from both techniques were similar, LiSAW showed distinctive advantage as it did not encounter the challenges of the nanostructured roughness. For thinner films, the advantages of LiSAW are even greater since the technique naturally accounts for substrate influence.

The results of this work have large implications towards emerging thin film energy materials. As evolving knowledge in this field continues to drive films in the direction of more complex structures, compositions, and geometries, new methodologies for mechanical characterization will inevitably be required. The robustness and performance of materials are frequently linked to mechanical properties, thus the ability to characterize mechanical behavior is critical in development. LiSAW is a promising tool for this application since it has shown capability on complex films. It also has great utility and versatility since it can selectively target the more difficult properties such as density and Poisson's ratio. As shown in this paper, the intricacy of future energy materials will undoubtedly stress current mechanical characterization techniques and the spectrum of film tests will undoubtedly benefit from the proficiencies afforded by LiSAW. Specific to electrode materials, it is hoped that the technique can be applied to many more emerging electrode thin films such that a deeper understanding of the relationship between mechanical properties and electrode performance can be discovered. 


\section{Acknowledgements}

G. Chow and J. Wang would like to acknowledge the financial support from National Science Foundation (CAREER award \#0935758).

\section{References:}

1. Holmes CF (2001) The role of lithium batteries in modem health care. J Power Sources 978:739-741

2. Whittingham MS (2012) History, Evolution, and Future Status of Energy Storage. P leee 100:1518-1534

3. Ji LW, Lin Z, Alcoutlabi M, Zhang XW (2011) Recent developments in nanostructured anode materials for rechargeable lithium-ion batteries. Energ Environ Sci 4 (8):2682-2699

4. Ellis BL, Lee KT, Nazar LF (2010) Positive Electrode Materials for Li-Ion and Li-Batteries. Chem Mater $22(3): 691-714$

5. Liu DW, Cao GZ (2010) Engineering nanostructured electrodes and fabrication of film electrodes for efficient lithium ion intercalation. Energ Environ Sci 3 (9):1218-1237

6. Song MK, Park S, Alamgir FM, Cho J, Liu ML (2011) Nanostructured electrodes for lithium-ion and lithium-air batteries: the latest developments, challenges, and perspectives. Mat Sci Eng R 72 (11):203-252

7. Zhang QF, Uchaker E, Candelaria SL, Cao GZ (2013) Nanomaterials for energy conversion and storage. Chem Soc Rev 42 (7):3127-3171

8. Vu A, Qian YQ, Stein A (2012) Porous Electrode Materials for Lithium-Ion Batteries - How to Prepare Them and What Makes Them Special. Adv Energy Mater 2 (9):1056-1085

9. Hayner CM, Zhao X, Kung HH (2012) Materials for Rechargeable Lithium-lon Batteries. Annu Rev Chem Biomol 3:445-471

10. Reimers JN, Dahn JR (1992) Electrochemical and In Situ X-Ray Diffraction Studies of Lithium Intercalation in Li x CoO2. Journal of The Electrochemical Society 139 (8):2091-2097

11. Meethong N, Huang HYS, Speakman SA, Carter WC, Chiang YM (2007) Strain accommodation during phase transformations in olivine-based cathodes as a materials selection criterion for high-power rechargeable batteries. Adv Funct Mater 17 (7):11151123

12. Cabana J, Monconduit L, Larcher D, Palacin MR (2010) Beyond Intercalation-Based Li-Ion Batteries: The State of the Art and Challenges of Electrode Materials Reacting Through Conversion Reactions. Adv Mater 22 (35):E170-E192

13. Ebner M, Marone F, Stampanoni M, Wood V (2013) Visualization and Quantification of Electrochemical and Mechanical Degradation in Li lon Batteries. Science 342 (6159):716720

14. Qu M, Woodford WH, Maloney JM, Carter WC, Chiang Y-M, Van Vliet KJ (2012) Nanomechanical Quantification of Elastic, Plastic, and Fracture Properties of LiCoO2. Adv Energy Mater 2 (8):940-944 
15. Ramdon S, Bhushan B (2014) Nanomechanical characterization and mechanical integrity of unaged and aged Li-ion battery cathodes. J Power Sources 246:219-224

16. Zhu J, Zeng KY, Lu L (2013) Cycling Effect on Morphological and Interfacial Properties of RuO2 Anode Film in Thin-Film Lithium Ion Microbatteries. Metall Mater Trans A 44A:2634

17. Li H, Vlassak JJ (2009) Determining the elastic modulus and hardness of an ultra-thin film on a substrate using nanoindentation. J Mater Res 24 (3):1114-1126

18. Hess $P$ (1996) Laser diagnostics of mechanical and elastic properties of silicon and carbon films. Applied Surface Science 106:429-437

19. Schneider D, Tucker MD (1996) Non-destructive characterization and evaluation of thin films by laser-induced ultrasonic surface waves. Thin Solid Films 290-291:305-311

20. Xiao X, Shan XM, Kayaba Y, Kohmura K, Tanaka H, Kikkawa T (2011) Young's modulus evaluation by SAWs for porous silica low-k film with cesium doping. Microelectron Eng 88 (5):666-670

21. Farnell GW, Adler EL (1972) Types and properties of surface waves. In: Mason WP, Thurston RN (eds) Physical Acoustics, vol 9. Academic Press, New York,

22. Cote R, Van der Donck T, Celis JP, Glorieux C (2009) Surface acoustic wave characterization of a thin, rough polymer film. Thin Solid Films 517 (8):2697-2701

23. Nazri GA, Pistoia G (2009) Lithium Batteries: Science and Technology. Springer,

24. Ding N, Feng X, Liu S, Xu J, Fang X, Lieberwirth I, Chen C (2009) High capacity and excellent cyclability of vanadium (IV) oxide in lithium battery applications. Electrochem Commun 11 (3):538-541

25. Liu YY, Clark M, Zhang QF, Yu DM, Liu DW, Liu J, Cao GZ (2011) V2O5 Nano-Electrodes with High Power and Energy Densities for Thin Film Li-lon Batteries. Adv Energy Mater 1 (2):194-202

26. Liu YY, Li JG, Zhang QF, Zhou N, Uchaker E, Cao GZ (2011) Porous nanostructured V2O5 film electrode with excellent Li-ion intercalation properties. Electrochem Commun 13 (11):1276-1279

27. Pomerantseva E, Gerasopoulos K, Chen XY, Rubloff G, Ghodssi R (2012) Electrochemical performance of the nanostructured biotemplated V2O5 cathode for lithium-ion batteries. J Power Sources 206:282-287

28. Augustyn V, Dunn B (2010) Vanadium oxide aerogels: Nanostructured materials for enhanced energy storage. Cr Chim 13 (1-2):130-141

29. Oliver WC, Pharr GM (1992) An Improved Technique for Determining Hardness and ElasticModulus Using Load and Displacement Sensing Indentation Experiments. Journal of Materials Research 7 (6):1564-1583

30. Chow G, Miller P, Wang J (2014) Correlation Between Laser-Induced Surface Acoustic Waves and Nanoindentation on Elastic Modulus Measurement of a Nanoporous Zeolite Thin Film. Experimental Mechanics

31. Xiao X, You X (2006) Numerical study on surface acoustic wave method for determining Young's modulus of low-k films involved in multi-layered structures. Applied Surface Science 253 (5):2958-2963

32. National Bureau of Standards US (1959) Circ. 539., vol 8. 
33. Cocciantelli JM, Doumerc JP, Pouchard M, Broussely M, Labat J (1991) Crystal chemistry of electrochemically inserted LixV2O5. Journal of Power Sources 34 (2):103-111

34. Braithwaite JS, Catlow CRA, Gale JD, Harding JH (1999) Lithium Intercalation into Vanadium Pentoxide: a Theoretical Study. Chemistry of Materials 11 (8):1990-1998

35. Delmas C, Brèthes S, Ménétrier M (1991) $\omega$-LixV2O5 - a new electrode material for rechargeable lithium batteries. Journal of Power Sources 34 (2):113-118

36. Galy J (1992) Vanadium pentoxide and vanadium oxide bronzes-Structural chemistry of single (S) and double (D) layer MxV2O5 phases. Journal of Solid State Chemistry 100 (2):229-245

37. B.V. Ratnakumar, M.C. Smart, Surampudi S (2001) Effects of SEI on the kinetics of lithium intercalation. J Power Sources 97-98:137-139

38. Keyes RW (1982) Device Implications of the Electronic Effect in the Elastic Constants of Silicon. Sonics and Ultrasonics, IEEE Transactions on 29 (2):99-103

39. Serway RA, Vuille C, Faughn JS (2008) College physics. vol v. 10. BROOKS COLE Publishing Company,

40. Wang Y, Cao G (2008) Developments in Nanostructured Cathode Materials for HighPerformance Lithium-Ion Batteries. Adv Mater 20 (12):2251-2269

41. Johnson MC, Wang JL, Li ZJ, Lew CA, Yan YS (2007) Effect of calcination and polycrystallinity on mechanical properties of nanoporous MFI zeolites. Materials Science and Engineering a-Structural Materials Properties Microstructure and Processing 456 (12):58-63

42. Lu X, Xiao P, Li H (2012) Effect of densification distribution on the Young's modulus of porous coatings after nano-indentation. Acta Metall Sin 25 (5):383-390 